\title{
Primary central nervous system lymphoma: relevant strategies for the initial management
} Linfoma primário do sistema nervoso central: estratégias relevantes na conduta inicial Suzana M. F. Malheiros

MD, PhD; Department of Neurology and Neurosurgery, Universidade Federal de São Paulo (UNIFESP); Neuro-oncology Program,

Hospiltal Israelita Albert Einstein, São Paulo SP, Brazil.

\section{Correspondence}

Suzana M. F. Malheiros Departamento de Neurologia e

Neurocirurgia, UNIFESP

Rua Botucatu 740

04023-900 São Paulo SP - Brasil

E-mail:smfmalheiros@gmail.com

\section{Conflict of interest}

There is no conflict of interest to declare.

Received 20 February 2013 Accepted 27 February 2013
$\mathrm{P}$ rimary central nervous system (CNS) lymphoma (PCNSL) is a relatively uncommon and aggressive extranodal non-Hodgkin lymphoma arising exclusively in the CNS that quite often presents a diagnostic challenge for several medical specialties, particularly for neurologists and neurosurgeons.

Diffuse large B-cell lymphoma comprises approximately 90\% of PCNSL. However, its prognosis is usually poorer than other extranodal diffuse large B-cell lymphomas, and therapeutic results are still disappointing. The limited available data about the molecular biology and pathophysiology of PCNSL precludes further advances in the development of new therapeutic approaches ${ }^{1}$.

PCNSL usually presents with a rapid onset in a variety of clinical syndromes including progressive encephalopathy or focal neurological deficits. Brain imaging often demonstrates enhancing lesions mimicking primary brain tumor, CNS infection, demyelinating disease and autoimmune or paraneoplastic syndromes. Both clinical presentation and neuroimaging features may be drastically improved with corticosteroid use, making the high index of suspicious a crucial step in the management of this condition.

The diagnostic evaluation of suspected PCNSL aims to confirm the diagnosis and define the extent of the disease besides excluding systemic involvement. Although there is little consensus, the systematic assessment of the available imaging, laboratory tests and biopsy targets can guide the evaluation and avoid unnecessary testing or delays in diagnosis.

Recently, Scott et al. ${ }^{2}$ conducted a review of the literature and proposed a systematic approach to the diagnosis of suspected PCNSL. The complete workout should include laboratory tests (complete blood count (CBC), HIV antibody, serum protein electrophoresis); neuroimaging (Magnetic Resonance Imaging (MRI); cerebrospinal fluid (CSF), if neuroimaging shows no risk of herniation; ophthalmologic examination; computed tomography (CT) or FDG-PET of the chest, abdomen and pelvis; and testicular ultrasonography (in males, especially $>60$ years of age). Ideally, once the diagnosis is suspected, the patient should not receive corticosteroids and should undergo a stereotactic confirmatory biopsy.

Methotrexate-based chemotherapy in different regimens is the basis of PCNSL treatment, usually followed by consolidation radiation therapy. Although chemoradiation has been the standard treatment for PCNSL patients for many years, the high frequency of neurotoxicity associated with this regimen remains challenging ${ }^{1}$. New therapeutic strategies are highly desirable to improve disease control and prevent neurotoxicity.

In this issue of Arquivos de Neuro-Psiquiatria, Perini and colleagues ${ }^{3}$ present a practical and useful review focused on the main strategies in the initial management of PCNSL. Some key points are emphasized:

1. The relatively low incidence and the dismal prognosis of PCNSL.

2. The importance of differentiating primary CNS lymphoma from secondary CNS lymphoma.

3. The differential diagnosis and the so called "vanishing tumor" syndrome.

4. The diagnosis confirmation preferably based on stereotactic biopsy rather than surgical resection.

5. The differences between the management of PCNSL and systemic lymphoma.

6 . The pros and cons of radiation therapy. 
PCNSL is a complex disease that requires a multidisciplinary approach. Neurologists and neurosurgeons are often the primary care physicians of these patients and must be aware of this challenging condition to avoid unnecessary delays in diagnosis that might compromise clinical outcome.

\section{References}

1. Brastianos PK, Batchelor TT. Primary central nervous system lymphoma: overview of current treatment strategies. Hematol Oncol Clin N Am 2012;26:897-916.

2. Scott BJ, Douglas VC, Tihan T, Rubenstein JL, Josephson SA. A systematic approach to the diagnosis of suspected central nervous system lymphoma. JAMA Neurol 2013; ahead of print.
3. PeriniGF, Campregher PV,Santos FPS, Hamerschlak N. Primary central nervous system lymphoma: what a neurologist/neurosurgeon should know? Arq Neuropsiquiatria 2013;71:254-257. 